\title{
The effect of medium composition on the production of sophorolipids and the tensiometric properties by Starmerella bombicola MTCC 1910
}

\author{
Nagarajan Vedaraman', Narayana Murthy Venkatesh ${ }^{2}$ \\ ${ }^{1}$ Chemical Engineering Division, Central Leather Research Institute, Chennai - 600020, India, \\ e-mail: nvedaraman@yahoo.co.in \\ ${ }^{2}$ Department of Chemical Engineering, St. Joseph's College of Engineering, Chennai - 600119, India, \\ e-mail: chemvenkatesh@gmail.com
}

\begin{abstract}
Starmerella bombicola a teleomorph of Candida bombicola is capable of producing extracellular secondary metabolites known as sophorolipids. In the present work the performance of Starmerella in producing sophorolipids, with standard medium ingredients glucose, sunflower oil, yeast extract and urea was studied. The quantities of different medium ingredients were optimized to maximize the production of sophorolipids. Variation in tensiometric properties like surface tension and interfacial tension during the incubation period were also reported. The optimized mixed substrate composition was found to be $200 \mathrm{~g} / \mathrm{l}$, containing equal amounts of glucose and sunflower oil, $4 \mathrm{~g} / \mathrm{l}$ of yeast extract and $0.6 \mathrm{~g} / \mathrm{l}$ of urea. With the optimized substrate composition $38.6 \mathrm{~g} / \mathrm{l}$ of sophorolipids was obtained. The minimum surface tension produced by the culture free cell broth was $36.2 \mathrm{mN} / \mathrm{m}$. Increasing the temperature from $25^{\circ} \mathrm{C}$ to $35^{\circ} \mathrm{C}$ has shown adverse effects on sophorolipids production.
\end{abstract}

Keywords: Biosurfactant, Lipidic substrates, Optimization, Sophorolipids, Starmerella bombicola, Tensiometric properties.

\section{INTRODUCTION}

Surfactants are defined as substrates that absorb to and alter the conditions prevailing at interfaces ${ }^{1}$. They reduce the free energy of the system by replacing the bulk molecules of higher energy at an interface. The effectiveness of a surfactant is determined by its ability to lower the surface tension, which is a measure of the surface free energy per unit area required to bring a molecule from the bulk phase to the surface ${ }^{2}$. The majority of the chemical surfactants are environmentally objectionable compounds. The increased environmental awareness and strict legislation has made the environmental compatibility of surfactants an important factor in their application for various uses.

Biosurfactants like glycolipids, rhamnolipids that are environmental friendly, non-toxic and active over a wide range of temperature and $\mathrm{pH}$ can be produced with reasonable yields by some microorganisms. One class of the biosurfactants that are currently receiving industrial consideration is the glycolipids. They are made up of a disaccharide sophorose (2-O-ß3-D-glucopyranosyl- 3 -D-glucopyranose) linked to a hydroxy fatty acyl moiety by a glycosidic bond between the 1' -hydroxy group of the sophorose sugar and the $\omega$ or $\omega-1$ carbon of the fatty acid. The acid function can be either free or esterified to the 4"- hydroxyl group of the sophorose, forming a monocyclic lactone. Typically the 6'- and 6"- hydroxy groups of the sophorose are acetylated and the fatty acid chain length varies between 16 and 18 carbons ${ }^{3}$. Sophorolipids are produced as complex mixtures containing both the free acid and lactone forms. These mixtures are typically brown oils, which are viscous and denser than water ${ }^{4}$. One of the perceived benefits of sophorolipids to the producing organisms is to help access and utilize lipophilic substrates ${ }^{5}$. The extracellular production of glycolipids was first studied and reported in the year 1961 by Candida species ${ }^{6}$. The culture medium constituents were glucose, yeast extract and urea. In later studies, different authors tested various production medium compositions using different hydrophilic and lipophilic carbon sources like oils and fatty acids which gave significantly higher yields of biosurfactants. For secondary metabolites like biosurfactants that are composed of moieties of significantly different oxidation states, production from a single carbon source is unfavorable. With mixed carbon sources, considerable improvements in the specific production rate and the final broth concentration may be achieved. There is a considerable reduction in the adenosine tri phosphate (ATP) demand for sophorolipids biosynthesis if a mixture of carbohydrate and lipidic source is used ${ }^{7}$. Yeast extract is considered to be essential for both cell growth and sophorolipids formation, and its concentration is critical in the culture medium, as sophorolipids synthesis is associated with nitrogen starvation. It is suggested that formation of glycolipids is some sort of overflow metabolism, by means of extracellular storage material. The most favorable concentration of yeast extract to be used still remains unclear ${ }^{8}$. There is a mixed opinion among the researchers whether or not to employ urea as secondary nitrogenous source. The use of urea as an input is believed to help in lipogenesis, conversion of glucose to fatty acids and increases the production of sophorolipids by yeast.

Starmerella bombicola is a less studied teleomorph of Candida bombicola whose ability to produce sophorolipids is not fully explored. In the present optimization studies Starmerella bombicola was grown in a shake flask with mixed substrate containing glucose, sunflower oil, yeast extract and urea in various proportions for seven days, to maximize the production of sophorolipids. The biomass growth patterns and variation in tensiometric properties of the cell free culture broth were reported with the optimized medium composition. 


\section{MATERIALS AND METHODS}

\section{Microorganism and Media}

Pure lyophilized strain of Starmerella bombicola MTCC 1910 was procured from Institute of Microbial Technology, Chandigarh, India and revived as per the specifications of the supplier. This was maintained in agar slants and transferred at regular intervals. Except sunflower oil, glucose, yeast extract and urea were of analytical reagent grade and procured from Himedia chemicals, India. The yeast extract used had $8-12 \%$ of the total nitrogen content and $3-5.2 \%$ of amino nitrogen content.

The medium always contained glucose, sunflower oil, yeast extract and urea but the concentrations of these nutrients varied in each run depending on the variables being studied. To study the effect of lipidic substrate on the production of sophorolipids and to arrive at the right proportion of carbohydrate and lipidic substrates, batches containing varying amounts of glucose and sunflower oil were employed along with $10 \mathrm{~g} / \mathrm{l}$ yeast extract and $1 \mathrm{~g} / \mathrm{l}$ urea. To quantify the right amount of the mixed substrate in the culture medium, glucose and sunflower oil both were put together in equal proportions at 5\%, 10\%, 15\%, $20 \%$ and $25 \%$ were employed with $10 \mathrm{~g} / \mathrm{l}$ yeast extract and $1 \mathrm{~g} / \mathrm{l}$ urea. The influence of the yeast extract was studied in $100 \mathrm{~g} / \mathrm{l}$ of glucose and $100 \mathrm{~g} / \mathrm{l}$ sunflower oil with different concentrations of the yeast extract from 0 to $10 \mathrm{~g} / \mathrm{l}$ as reported by other researchers ${ }^{\mathbf{9}}$. Similarly to study the effect of urea, $100 \mathrm{~g} / \mathrm{l}$ of glucose, $100 \mathrm{~g} / \mathrm{l}$ of sunflower oil and $4 \mathrm{~g} / \mathrm{L}$ of yeast extract with different concentration of urea, from 0 to $1 \mathrm{~g} / \mathrm{l}$ were employed. Finally the effect of temperature on the production of sophorolipids was examined at $25^{\circ}, 30^{\circ}$ and $35^{\circ} \mathrm{C}$ with the optimized quantities of the substrates.

\section{Shake flask experiments}

Seed culture was developed from frozen Starmerella in the $250 \mathrm{ml}$ Erlenmeyer flasks with $50 \mathrm{ml}$ medium, consisting of $5 \mathrm{~g}$ glucose, $0.5 \mathrm{~g}$ yeast extract and $0.05 \mathrm{~g}$ urea by keeping it over a rotary shaker at $200 \mathrm{rpm}$ for 48 hours. Shake flask experiments were conducted in the 0.5 liter Erlenmeyer flasks on a rotary shaker with the $100 \mathrm{ml}$ medium containing different concentrations in each run depending on the variables being studied. The sterile fermentation medium was inoculated with $10 \%$ (v/v) seed culture to begin the sophorolipids production. The experiments were conducted at $30^{\circ} \mathrm{C}$ and $170 \mathrm{rpm}$, with initial $\mathrm{pH}$ adjustment to 6.0 , for seven days.

\section{Determination of Biomass}

The biomass formed was monitored by the estimation of dry cell weight after the removal of hydrophobic substrates as per the reported method ${ }^{\mathbf{1 0}}$. Briefly $1 \mathrm{ml}$ aliquot from the fermentation broth was centrifuged to obtain a cell pellet. The supernatant was discarded and the cell pellet was washed with water to remove residual sugars and medium components. The pellet was then washed with $1 \mathrm{ml}$ of ethyl acetate to remove sophorolipids and other hydrophobic substances. The washed cells were centrifuged to obtain a pellet, which was dried in vacuum to a constant weight, and expressed in terms of $\mathrm{g} / \mathrm{l}$.

\section{Estimation of Tensiometric Properties}

The measurement of the surface tension was carried out on the cell free culture broth obtained by centrifuging the cultures at $4000 \mathrm{rpm}$ for $20 \mathrm{~min}$ as per the standard procedures, by the du Nouy-ring method using Surface tension Balance - Interfacial tensiometer with $4 \mathrm{~cm}$ platinum ring as per Indian standards methods (IS) 6104 and American Society for Testing and Materials (ASTM) D 971 specifications, at room temperature ${ }^{\mathbf{1 1}}$. A $30 \mathrm{ml}$ of cell free culture broth was taken in a glass vessel $(50 \mathrm{ml})$ and was placed on the tensiometer. A platinum wire ring was placed into the solution and then slowly pulled through the liquid-air interface. Stabilization was allowed to occur until a standard deviation of 10 successive measurements was less than $0.4 \mathrm{mN} / \mathrm{m}$. Between each measurement, the platinum ring was rinsed with water and acetone and allowed to dry. The minimum interfacial tension against $\mathrm{n}$-hexadecane was determined.

\section{Recovery and quantification of biosurfactant}

The volume of the entire culture mixture after fermentation was measured and centrifuged at $10,000 \mathrm{rpm}$ at $4^{\circ} \mathrm{C}$ for $20 \mathrm{~min}$. The supernatant was extracted twice with equal volumes of ethyl acetate. The extracts were filtered though Whatman No 2 filter paper and the remaining solids were washed again with ethyl acetate to maximize the recovery ${ }^{12}$. The combined ethyl acetate fractions containing the sophorolipids were concentrated by rotary vacuum evaporation. It was then washed with hexane to remove lipidic impurities, and accurately weighed to obtain the final product.

The structural classes present in the sophorolipids product were evaluated using thin layer chromatography (TLC). The components were identified by comparison of the calculated retention factor ( $\mathrm{Rf}$ ) values with those reported elsewhere using the same TLC developing solvent system consisting of chloroform/methanol/water in the ratio 65:15:20 by $\mathrm{vol}^{4}$. After development, the plate was dried and sprayed with $\alpha$-naphthol (98\%) to detect the monosophorolipids. The sophorolipids classes were identified by comparison with standards.

\section{RESULTS AND DISCUSSION}

\section{Effect of media Composition on the growth and sophorolipids Production}

Table 1 shows the final biomass, amounts of sophorolipids and tensiometric properties of the cell free culture broths. The yeast is able to assimilate the fatty acid directly from the production medium or to synthesize it from any carbon source ${ }^{\mathbf{1 3}}$. In the literature available, the right proportions of carbohydrate and lipid sources were varying from 1:0.4 to $1: 1.5^{\mathbf{1 4}-\mathbf{1 6}}$. To arrive at the right proportion of carbohydrate and lipidic source, medium composition consisting of varying amounts of glucose and oil were employed. Mixed substrate consisting of $100 \mathrm{~g} / \mathrm{l}$ of glucose and $100 \mathrm{~g} / \mathrm{l}$ of sunflower oil gave maximum production. Initially, Starmerella bombicola grows by consuming glucose, and because of the stress created by the presence of sunflower oil it produces extracellular product sophorolipids. One of the perceived benefits of sophorolipids for the producing organism is to 
Table 1. The influence of the composition of media culture

\begin{tabular}{|c|c|c|c|c|}
\hline $\begin{array}{c}\text { Amount of glucose } \\
\mathrm{g} / \mathrm{l}\end{array}$ & $\begin{array}{c}\text { Amount of } \\
\text { sunflower oil } \mathrm{g} / \mathrm{l}\end{array}$ & $\begin{array}{c}\text { Biomass } \\
\text { formation } \\
\mathrm{g} / \mathrm{l}\end{array}$ & Surface Tension $\mathrm{mN} / \mathrm{m}$ & $\begin{array}{c}\text { Sophorolipids } \\
\mathrm{g} / \mathrm{l}\end{array}$ \\
\hline 100 & 0 & 18.5 & 44 & 22.12 \\
\hline 100 & 50 & 21.4 & 38 & 31.8 \\
\hline 100 & 100 & 27.5 & 37 & 32.14 \\
\hline 50 & 100 & 20.8 & 37 & 17.19 \\
\hline 0 & 100 & 22.3 & 52 & Traces \\
\hline
\end{tabular}

help access and utilize lipophilic substrates as reported in literature ${ }^{3}$.

Comparison of the $\mathrm{R}_{\mathrm{f}}$ values found from thin layer chromatography revealed that two prominent types of sophorolipids are present in the product mixture, SL-7 and SL-1. SL-7 is 1', 4"-Lactone 6'6'-diacetate with unsaturated C18:1 fatty acid moiety, where as SL-1 is 1', 4"Lactone 6'6'-diacetate with saturated C18 fatty acid moiety.

\section{Effect of the amount of mixed substrate on the production of sophorolipids}

To establish the effect of the amount of combined carbon source, in the culture medium, varying amounts of equal proportion of glucose and sunflower oil constituting $5 \%$ to $25 \%$ were employed. Fig. 1 shows the effect of the amount of the mixed substrate in the medium, on the production of biomass and sophorolipids. Any amount higher than 20\% (100 g/l glucose and $100 \mathrm{~g} / \mathrm{l}$ sunflower oil) has shown negative effects on sophorolipids production, due to substrate inhibition. Furthermore oxygen transfer rate is very critical for the growth and production of sophorolipids. With higher concentrations of the substrate, due to improper oxygen transfer rates, both the biomass and sophorolipids production gets affected.

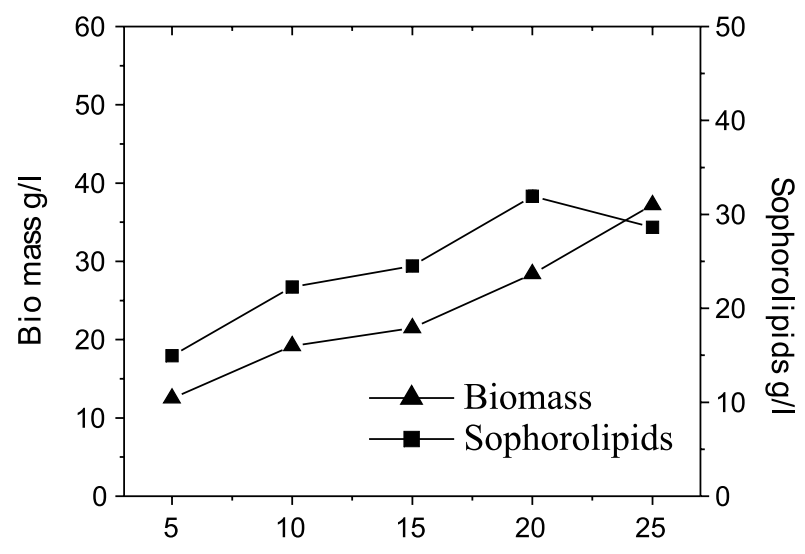

Mixed substrate quntity (in \%) in medium culutre

Figure 1. Biomass and sophorolipids amount with percentage mixed substrate in the culture

\section{Effect of the amount of yeast extract}

Sophorolipids production occurs after growth when the nitrogen source yeast extract is exhausted. Hence the concentration of yeast extract plays a vital role. Fig. 2 shows the effect of yeast extract in the culture medium. Different yeast extract concentrations from 0 to $10 \mathrm{~g} / \mathrm{l}$ were employed. As the yeast extract concentration increased, biomass formation has increased and sophorolipids production also increased up to $4 \mathrm{~g} / \mathrm{l}$ of yeast extract, however a further increase in yeast extract led to the reduction in the sophorolipids production. Tensiometric studies on the cell free culture broths have shown surface tension of $37-38 \mathrm{mN} / \mathrm{m}$ and interfacial tension with $\mathrm{n}$-hexadecane for all the samples were 2.8 $\mathrm{mN} / \mathrm{m}$. At higher yeast extract concentrations sophorolipids production decreases due to depleted carbon sources.

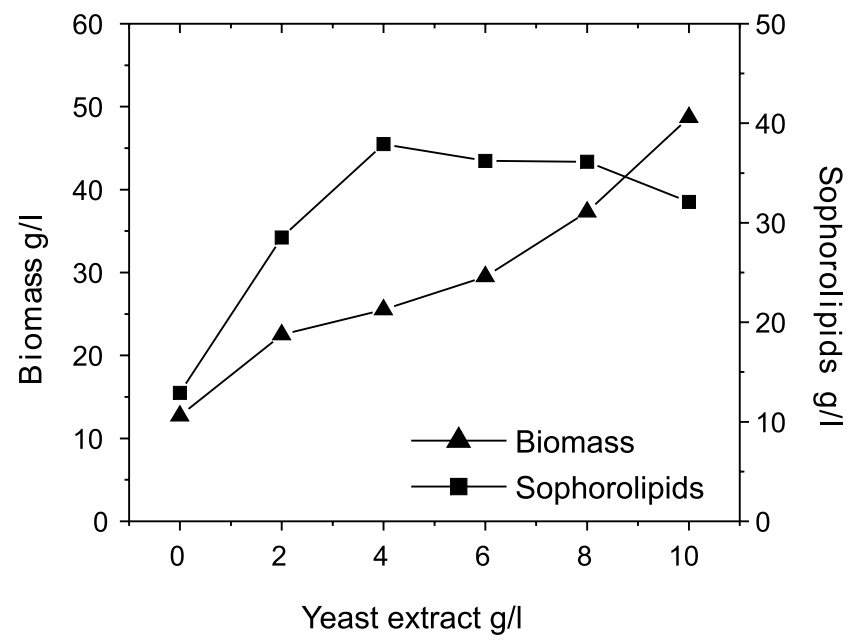

Figure 2. The influence of yeast extract on biomass and sophorolipids

Effect of Secondary Nitrogen source Urea on the production of Sophorolipids

Several researchers have used different amounts of urea as secondary nitrogenous source and others have not ${ }^{3,4,9,17}$. The maximum amount of urea used, reported in the literature is $1 \mathrm{~g} / \mathrm{l}$. Fig. 3. shows the effect of urea on the production of sophorolipids. The use of urea as an input helps in lipogenesis, and increases the formation of sophorolipids. The optimum urea concentration was found to be $0.6 \mathrm{~g} / \mathrm{l}$.

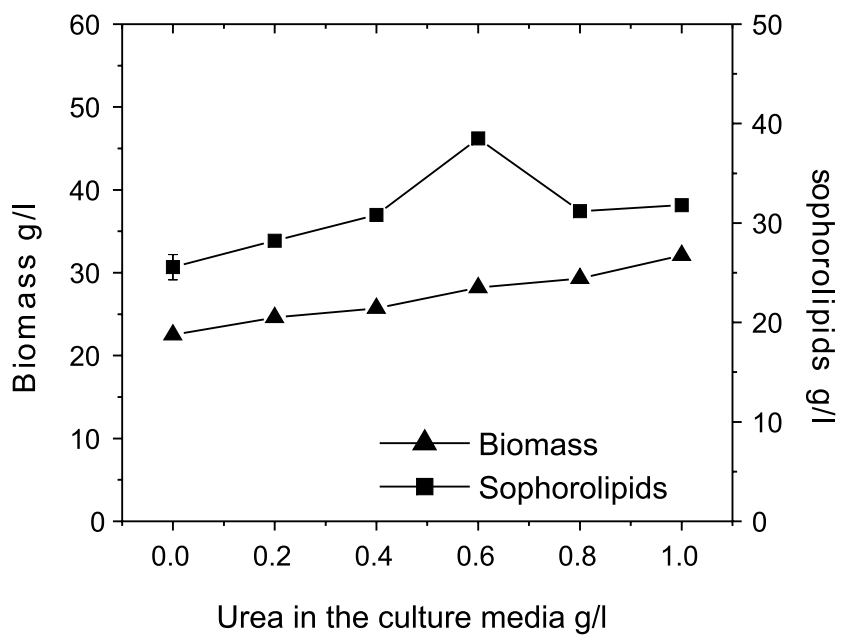

Figure 3. The influence of urea on biomass and sophorolipids production 


\section{Effect of Temperature on the production of Glycolipids}

Fig. 4 shows the effect of temperature on the production of sophorolipids. Temperature has got adverse effect on the production of sophorolipids. Higher temperatures favored the effective consumption of substrate, which resulted in healthy biomass formation but the product formation got decreased. The temperature between $25^{\circ} \mathrm{C}-30^{\circ} \mathrm{C}$ was found to be optimum for the production of sophorolipids.

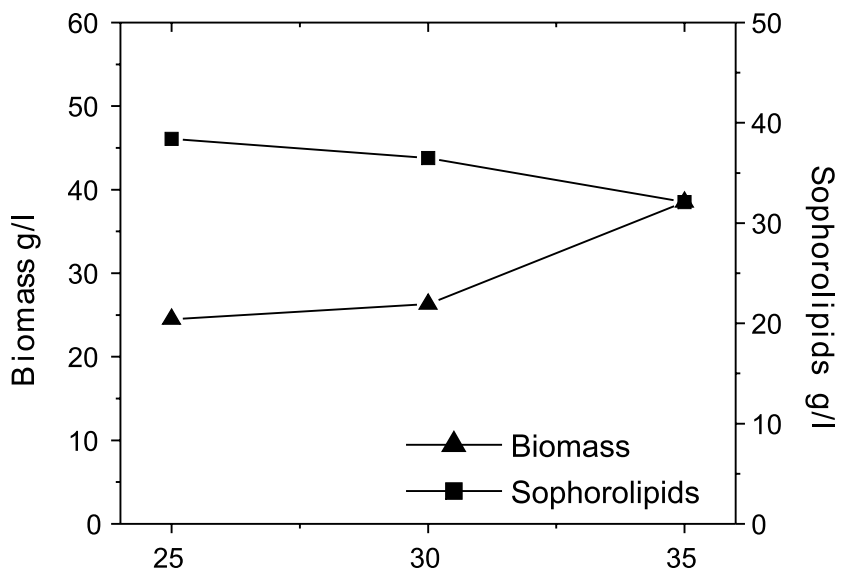

Temperature ${ }^{\circ} \mathrm{C}$

Figure 4. The effect of temperature on biomass and sophorolipids production

Biomass growth and Tensiometric Properties during the incubation period with optimized culture medium

Eight parallel experiments were conducted at optimal conditions, at $25^{\circ} \mathrm{C}$ with $100 \mathrm{~g} / \mathrm{l}$ of glucose, $100 \mathrm{~g} / \mathrm{l}$ of sunflower oil, $4 \mathrm{~g} / \mathrm{l}$ of yeast extract and $0.6 \mathrm{~g} / \mathrm{l}$ of urea, to understand the sophorolipids production, for seven days. Each set was withdrawn every day, to determine the progressive variation in the biomass growth, the amount of sophorolipids produced and tensiometric properties. The experimental results are shown in Figure 5 and 6.

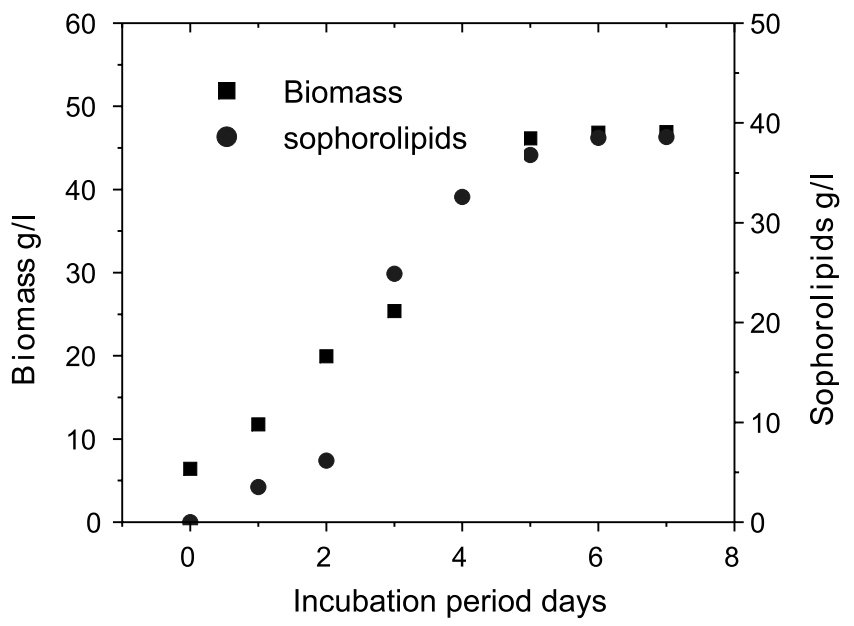

Figure 5. The effect of fermentation time on biomass and sophorolipids production

The objective of this experiment was to observe the stage at which sophorolipids were produced and released by the yeast. It is very clear from Figure 5 and 6 that the maximum amount of sophorolipids was produced in the late exponential growth period, that is the $3^{\text {rd }}$ and $4^{\text {th }}$ day. A further increase in experimental duration to 5, 6 and 7

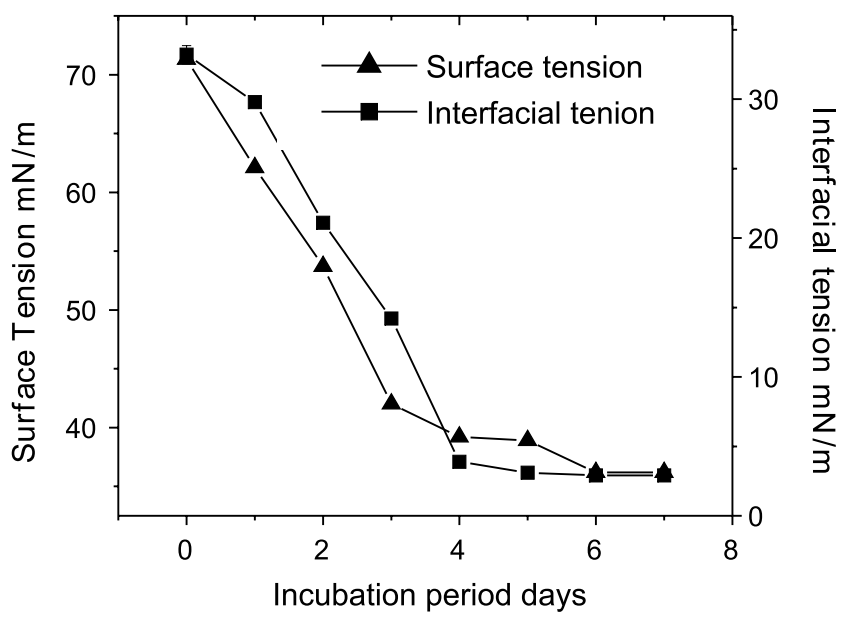

Figure 6. The effect of fermentation time on the tensiometric properties

days has shown a very slight increase in sophorolipids production. Hence the experiments were terminated on the seventh day. The optimum amount of sophorolipids obtained was found to be $38.6 \mathrm{~g} / \mathrm{l}$. The minimum surface tension of the cell free culture broth achieved was 36.2 $\mathrm{mN} / \mathrm{m}$ and interfacial tension with hexadecane was 2.9 $\mathrm{mN} / \mathrm{m}$.

\section{CONCLUSIONS}

Starmerella bombicola a relatively less studied teleomorph of Candida bombicola was grown in a shake flask with mixed substrate containing glucose and sunflower oil in different proportions to optimize the production of sophorolipids. To quantify the exact amount two nitrogenous sources, yeast extract and urea, a number of experiments were conducted with different amounts of these two nitrogenous sources. During the incubation time biomass was formed, sophorolipids produced and the tensiometric properties were monitored.

Under nitrogen starvation conditions, biomass formation reduces and sophorolipids production increases. It was found that an addition of yeast extract helped in sophorolipids production up to $4 \mathrm{~g} / \mathrm{l}$ beyond which there was no further increase. Higher yeast concentrations favored biomass formation, but sophorolipids production got reduced. Urea as a secondary nitrogen source had shown marginal increase on sophorolipids production and an optimum amount was found to be $0.6 \mathrm{~g} / \mathrm{l}$. The temperature showed an adverse effect on sophorolipids production, the optimum temperature was found to be $25^{\circ} \mathrm{C}$. The optimum conditions were found to be $100 \mathrm{~g} / \mathrm{l}$ of glucose, $100 \mathrm{~g} / \mathrm{l}$ of sunflower oil, $4 \mathrm{~g} / \mathrm{l}$ of yeast extract and $0.6 \mathrm{~g} / \mathrm{l}$ of urea, where a maximum quantity of $38.6 \mathrm{~g} / \mathrm{l}$ sophorolipids was obtained. It was confirmed that, the maximum amount of sophorolipids production by Starmerella bombicola is taking place during late exponential phase of growth.

\section{LITERATURE CITED}

1. Rosenberg, E. \& Ron E.Z. (1999). High molecular mass microbial surfactants. Appl. Microbiol. Biotechnol. 52, 154 162. DOI 10.1007/s002530051502.

2. Mulligan, C.N. (2005). Environmental applications for Biosurfactants, Environ. Pollut. 133, 183 - 198. DOI 10.1016/ j.envpol.2004.06.009. 
3. Inoue, S. \& Ito S. (1982) Sophorolipids form Torulopsis bombicola: Possible relation to alkane uptake. Appl. Environ. Microbiol. 43, 1278 - 1283.

4. Asmer, H.J., Lang, S., Wagner, F. \& Wray, V. (1988) Microbial production, structure elucidation and bioconversion of sophorose lipids. J Am Oil Chem Soc. 65, 1460 - 1466. DOI 10.1007/BF02898308.

5. Tulloch, A.P., Spenser, J.F.T. \& Gorin, P.A.J. (1962). The fermentation of long chain compounds by Torulopsis magnoliae. Can. Journal of Chem., 40, 1326 - 1338. DOI 10.1139/v62-203.

6. Gorin, P.A.J., Spenser, J.F.T. \& Tulloch, A.P. (1961) Hydroxy Fatty acid Glycosides of Sophorose from Torulopsis magnoliae. Can. Journal of Chem. 39, 846 - 855. DOI 10.1139/ v61-104.

7. Linton, J.D. (1991) Metabolite production and growth efficiency. Antonie van Leeuwenhoek. 60, 293 - 311. DOI 10.1007/BF00430371.

8. Inge, N.A., Bogaert, V., Saerens, K., Muynck, C.D., Develter, D., Soetaert, W. \& Vandamme, E. J. (2007) Microbial production and application of Sophorolipids. Appl. Microbiol Biotechnol. 76, 23 - 34. DOI 10.1007/s00253-0070988-7.

9. Casas, J.A. \& Garcia-Ochoa, F. (1999). Sophorolipid production by Candida bombicola: Medium composition and Culture methods. J. Biosci. Bioeng. 88, 488 - 494. DOI 10.1016/ S1389-1723(00)87664-4.

10. Zhou, Q.H., Klekner, V. \& Kosaric, N. (1992) Production of sophorose lipids by Torulopsis bombicola from Safflower Oil and Glucose. Appl. Environ. Microbiol. 47, 89 - 91. DOI 10.1007/BF02635883.

11. Surubbo, L.A., Farias, C.B.B. \& Campos-Takaki, G.M. (2007). Co Utilization of Canola Oil and Glucose on the production of a surfactant by Candida lipolytica. Curr. Microbiol. 54, 68 - 73. DOI 10.1007/s00284-006-0412-z.

12. Ashby, R.D., Nunez, A., Solaiman, D.K.Y. \& Foglia, T.A. (2005). Sophorolipid biosynthesis from a Biodiesel co product stream. J Am Oil Chem Soc. 82, 625 - 630. DOI 10.1007/s11746-005-1120-3.

13. Rau, U., Manzke, C. \& Wagner, F. (1996). Influence of substrate supply on the production of sophorose lipids by Candida bombicola ATCC 22214. Biotechnol. Lett., 18, 149 154. DOI 10.1007/BF00128670.

14. Felse, P.A., Shah, V., Chan, J., Rao, K.J. \& Gross, R.A. (2007). Sophorolipid biosynthesis by Candida bombicola from industrial fatty acid residues. Enzyme and Microb Technol. 40, 316 - 323. DOI 10.1016/j.enzmietec.2006.04.013.

15. Fleurackers, S.J.J. (2006). On the use of Waste frying oil in the synthesis of sophorolipids. Eur. J. Lipid. Sci. Technol. 108, 5 - 12. DOI 10.1002/ejlt.200500237.

16. Kim, Soo, H., Kim, Y. B., Lee, B. S. \& Kim, E.K. (2005) Sophorolipid Production by Candida bombicola ATCC 22214 from a Corn Oil processing byproduct. J. Microbiol. Biotechnol. $15,55-58$.

17. Cavalero, D.A. \& Cooper, D.G. (2003). The effect of medium composition on the structure and physical state of Sophorolipids produced by Candida bombicola ATCC 22214. J. Biotechnol., 103, 31 - 41. DOI 10.1016/S0168-1656(03)00067-1. 